\title{
A series of contexts relative to a matroid*
}

\author{
Hua Mao, Lanzhen Yang \\ (Department of Mathematics, Hebei University, Baoding 071002, China) \\ (Department of Mathematics, Hebei University, Baoding 071002, China)
}

\begin{abstract}
This paper presents some contexts relative to a matroid, which satisfy: all the closure operators derived from their Galois connections which to these contexts are the closure operator of the matroid. Using these results and ready-made algorithms for building up a concept lattice or an extent lattice, we believe that we may search out the class of all closed sets of a matroid, and meanwhile, create the construction of the dual of a matroid.
\end{abstract}

Keywords: context; closed set; matroid; Galois connection; closure operator 2000 AMSC 05B35; 06A15; 68R05

\section{Introduction}

The word matroid was coined by Whitney in 1935 (cf.[1]). There are many algorithms to construct matroids in the way of searching out the family of independent sets of a matroid (cf.[2-4]). However, it is well known that there are dozens of equivalent ways to define a matroid. For example, there is a definition to define a matroid with the family of closed sets (cf.[2]). The family of closed sets of a matroid plays an important role in matroid theory and produces a lot of results. The famous one is that Welsh in [2,Chapter 3] presents the relationship between matroids and geometric lattices. The relationship is relative to the family of all closed sets of matroids. Also, many results in [2] are associated with the family of closed sets of a matroid. All these results relative to the family of closed sets of a matroid demonstrate that it is necessary to search out all the closed sets of a matroid if we consider some properties of a matroid. But, up till now, according to our knowledge, there are only a few approaches directly or indirectly to search out all the closed sets of a matroid (cf.[5,6]). Hence, if we want to study on matroids deeply, then we may try to find out the other ideas to search out all the closed sets of a matroid. For this purpose, we may notice the following statements:

* This research is supported by National Natural Science Foundation of China (61572011)

(1.1) Concept lattices are a principal way to automatically derive an ontology from a collection of objects and their properties. The term was introduced by R.Wille (cf.[7]), and built on applied lattice and order theory that was developed by Birkhoff et al. (cf.[8]).

(1.2) Galois connections have applied in various mathematical theories (cf.[9-12]).

(1.3) For a context, there are many algorithms to search out all the concepts, and further, the concept lattices (cf.[13-19]).

(1.4) In [20], it presents the correspondent relationship between algebraic lattices and concept lattices. We may know [8] that the relationship between algebraic lattices and geometric lattices is that an algebraic lattice is geometric, but not vice versa. Welsh in [2] points out that for finite cases, up to isomorphism, there is a correspondent relationship between simple matroids and geometric lattices.

(1.5) Combining the above (1.1)-(1.3) and the relationship between matroids and lat- tices, we naturally ask a question: shall we use some already made-algorithms for construct- ing concept lattices to construct all the closed sets of a matroid?

(1.6) The three relationships in (1.4) also state that it is valuable to discuss the relation between contexts and matroids for finite status. Though, in [20], it deals with algebraic lattices in formal concept analysis, it does not provide an idea to discover a geometric lattice directly from formal concept analysis. That is to say, it does not provide an idea to discover a matroid from formal concept analysis. Even though, the reference [20] is still good and helpful in dealing with lattices and formal concept analysis.

Based on (1.1)-(1.6), to seek the answer of the above question in (1.5), we may think that the most important step is to work out a suitable context $(O, P, R)$ such that $\left\{X \subseteq O \mid\left(X, X^{\prime}\right) \in \operatorname{Gal}(O\right.$, $P, R)\}$ is exactly $\mathcal{F}$, where $\operatorname{Gal}(O, P, R)$ is the set of all the concepts of $(O, P, R)$ and $\mathscr{F}$ is the family of all closed sets of a given matroid on $O$.

This paper is to find some contexts satisfying $\left\{X \subseteq O \mid\left(X, X^{\prime}\right) \in \operatorname{Gal}(O, P, R)\right\}=\mathcal{F}$ for a matroid $M$ on $O$ with $\mathcal{F}$ as its family of closed sets. Using these contexts, it provides some applications 
in mathematical theories, especially, in finding out all the closed sets of $M$. Certainly, the question in (1.5) is answered. According to [7], we may describe that concept lattice is the central content in formal concept analysis. Thus, we may state that the results in this paper are helpful to the discussion on geometric lattices and formal con- cept analysis in our future.

The structure of this paper is as follows. In Section 2, it reviews some terminologies of Galois connections, some known notions and properties about concept lattices and lattice theory and some properties of matroid theory. Thereafter, Section 3 provides some contexts for a matroid. Section 4 discusses some applications for the contexts provided in Section 3.

We may indicate that though there are finite matroids and infinite matroids, a context is finite. Thus, we may suppose that all the discussions in this paper are finite.

\section{Preliminaries}

The following is to summarize the known facts of Galois connections, concept lattices, lattice theory and matroid theory that are needed later on. For more details, lattice theory is referred to $[2,8,10]$; concept lattice theory, please see [9,10]; matroid theory, please refer to [2].

Let $X$ be a set, and $\gamma(X)$ be the collection of all subsets of $X$.

Definition 1 (1)[11] Let $O$ and $P$ be sets. Let $R$ be a relation between $O$ and $P$; in symbols, $R \subseteq$ $O \times P$. For any subset $X$ of $O$, let

$K(X):=\{y \in P \mid \forall x \in X,(x, y) \in R\}$.

For any subset $Y$ of $P$, let

$L(Y):=\{x \in O \mid \forall y \in Y,(x, y) \in R\}$.

This pair $(K, L)$ of mappings is a Galois connection between $O$ and $P$.

A mapping $J: \varnothing(O) \rightarrow \varnothing(O)$ is a closure operator on $O$ if it satisfies the following conditions for all subsets $X$ and $Y$ of $O$ :

(s1) $X \subseteq J(X)$.

(s2) $X \subseteq Y \Rightarrow J(X) \Rightarrow J(Y)$. (s3) $J J(X)=J(X)$.

(2)[10,p.146\&9] An element $X \in \mathcal{O}(O)$ is called closed if $J(X)=X$.

The definitions of the Galois connection and closure operator in [9] are the same to that in [10] respectively. In fact, the correspondent definitions in Definition 1 are the same to that in $[9,10]$.

Lemma 1 [11] Let $O$ and $P$ be sets and let $K: \varnothing(O) \rightarrow \varnothing(P)$ and $L: \varnothing(P) \rightarrow \varnothing(O)$

be maps which form a Galois connection. Then the map $L K$ is a closure operator on $O$.

Next we review some needed notations and properties.

Definition 2 (1)[10,p.66\&9] A context is a triple $(O, P, R)$ where $O$ and $P$ are sets and $R \subseteq O \times P$. For $A \subseteq O$ and $B \subseteq P$, define

$A^{\prime}=\{m \in P \mid \forall g \in A,(g, m) \in R\}, B^{\prime}=\{g \in O \mid \forall m \in B,(g, m) \in R\}$.

A concept of $(O, P, R)$ is defined to be a pair $(A, B)$ where $A \subseteq O, B \subseteq P, A^{\prime}=B$ and

$B^{\prime}=A$. The extent of the concept $(A, B)$ is $A$. The set of all concepts of $(O, P, R)$ is denoted by $\operatorname{Gal}(O, P, R)$.

(2) $[2$, p.51\&8,10] A finite lattice is geometric if it is semimodular and every point is the join of atoms.

$[9$, p.20,Theorem 3] and [10,p.67,3.4] point out that there is a lattice construction gener- ated by $\operatorname{Gal}(O$, $P, R)$. This concept lattice is still denoted as $\operatorname{Gal}(O, P, R)$. Let $\bigotimes_{O}:=\left\{A \subseteq O \mid A^{\prime \prime}=A\right\}$.

Lemma 2 (1)[10,p.68] ( $\mathcal{B}_{O}, \subseteq$ ) is a lattice which is isomorphic to $\operatorname{Gal}(O, P, R)$. (2)[2,p.55\&8,10] Any interval of a geometric lattice is geometric.

The authors in $[9,10]$ indicate that in Definition 2, the operation ': $\varnothing(O) \rightarrow \varnothing(P)$ and the operation ${ }^{\prime}: \varnothing$ $(P) \rightarrow \varnothing(O)$ is a Galois connection for a context $(O, P, R)$ respectively. This states equivalently that in a context $(O, P, R)$, for $A \subseteq O$ and $B \subseteq P$, there are $A^{\prime}=K(A)$ and $B^{\prime}=L(B)$, where $(K, L)$ is defined as Definition 1.

According to Lemma 2 , we may call $(\varangle O, \subseteq)$ extent lattice for a context $(O, P, R)$, simply in notation $\varangle O$ if there is no confusion from the text.

Combining [10,p.159,7.2.6 \&9,11] with [10,pp.160-162\&9], we may express the following views:

(v1) a Galois connection $(K, L)$ between $O$ and $P$ is produced by a context $(O, P, R)$, and further by $\operatorname{Gal}(O, P, R)$.

Both $\operatorname{Gal}(O, P, R)$ and $(O, P, R)$ are produced by the same Galois connection $(K, L)$ 
between $O$ and $P$.

(v2) $\varangle_{O}=\Theta(L K)$, where $\Theta(L K)=\{X \subseteq O \mid L K(X)=X\}$.

We review the definitions and properties relative with a matroid which are best to our topic.

Definition 3 (1)[2,p.7] A finite matroid $M$ is a pair $(E, g)$, where $E$ is a finite set and

$\mathcal{\zeta}$ is a collection of subsets of $E$ (called independent sets) with the following properties: (i1) $\varnothing \in \mathcal{\zeta}$.

(i2) $X \subseteq Y \in \mathcal{G} \Rightarrow X \in \mathcal{G}$.

(i3) $A, B \in \mathcal{G}$ and $|A| \geq|B| \Rightarrow$ there exists $a \in A \backslash B$ satisfying $B \cup a \in \mathscr{\sigma}$. (2)[2,p.7] The rank function of $M$ is a function $\rho: \gamma(E) \rightarrow Z$ defined by

$\rho(A)=\max \{|X| \mid X \subseteq A, X \in \xi\},(A \subseteq E)$.

(3)[2,p.8] $A \subseteq E$ is closed of $M$ if for all $x \in E \backslash A, \rho(A \cup x)=\rho(A)+1$.

If for $x \in E$ and $A \subseteq E$, it has $\rho(A \cup x)=\rho(A)$, we say that $x$ depends on $A$. We define the closure operator of $M$ to be a function $\sigma: \varnothing(E) \rightarrow \varnothing(E)$ such that $\sigma(A)$ is the set of elements which depend on $A$.

(4)[2,p.38] A hyperplane of $M$ is a maximal proper closed subset of $E$.

Lemma 3 (1)[2,pp.8-9] A function $\sigma$ on $E$ is the closure operator of a matroid $M$ on a finite set $E$ if and only if $\sigma$ satisfies (s1)-(s3) and the following condition:

(s4) for $Y \subseteq E$ and $y, z \in E$, if $y \in \sigma(Y)$ but $y \in \sigma(Y \cup\{z\})$, then $z \in \sigma(Y \cup\{y\})$.

(2)[2,p.8] A function $\rho: \supset(E) \rightarrow \boldsymbol{Z}$ is the rank function of a matroid on $E$ if and only if for $X \subseteq E, y, z$ $\in E$ :

(r1) $\rho(\varnothing)=0$.

(r2) $\rho(X) \leq \rho(X \cup y) \leq \rho(X)+1$.

(r3) $\rho(X \cup y)=\rho(X \cup z)=\rho(X) \Rightarrow \rho(X \cup y \cup z)=\rho(X)$. (3)[2,p.22] If $X$ and $Y$ are closed sets of $M$, then so is $X \cap Y$.

(4)[2,p.51] $(\mathcal{F}, \subseteq)$ is a geometric lattice, where $\mathcal{F}$ is the family of closed sets of $M$.

By Lemma 1 and Lemma 3, the closure operator $\sigma$ of a matroid $M$ on $E$ is a closure operator on $E$, but not vice versa.

Let $M$ be a matroid on $E$ with $\sigma$ and $\rho$ as its closure operator and the rank function. Based on Definition 3 and Lemma 3 , in this paper, we denote $M$ as $(E, \sigma)$ and $(E, \rho)$ re- spectively if it does not cause the confusion.

In what follows, $M$ stands for a matroid on a finite set $E$ with $\rho, \sigma, \mathcal{F}, \mathcal{F}$ as its rank function, its closure operator, its family of closed sets and its class of independent sets.

Considered the above analysis and the results in [2], we may state easily the following properties for any $X \subseteq E$ and $y \in E$,

(I) $\rho(\sigma(X))=\rho(X)$.

(II) $\rho(\sigma(X) \cup y)=\rho(X \cup y)$. (III) $X \in \mathcal{\zeta} \Rightarrow|X|=\rho(X)$.

(IV) $\rho(\sigma(X) \cup y)=\rho(X \cup y)=\rho(B \cup y)$ for any base (i.e. a maximal independent set) $B$ of $X$.

(V) $\sigma(X \cup y)=\sigma(X) \Leftrightarrow y \in \sigma(X)$. (VI) $\sigma(\sigma(X) \cup y)=\sigma(X \cup y)$.

(VII) Let $B \subseteq X$. Then $B$ is a base of $X$ if and only if $\rho(X)=|B|$.

\section{Contexts}

In [20], it deals with more properties of algebraic lattices and obtains the relationships between algebraic lattices and formal concept lattices. These results make algebraic lat- tices apply in many parts such as formal concept analysis theory (cf.[20]). We may use the discussion line as in [20] to begin our discussion with matroids and contexts in this section First, for a matroid $M$ defined on a finite set, we provide some contexts corresponding to $M$ satisfying $\sigma=L K$, where $(K, L)$ is the Galois connections generated from any of contexts provided here. After that, the relationships among these contexts is dealt with.

Theorem 1 Let $R \subseteq O \times \varnothing(O)$ be defined as:

for any $x \in O$ and $Y \subseteq O,(x, Y) \in R \Leftrightarrow \rho(Y \cup x)=\rho(Y)$ and $\sigma(Y)=Y$.

If $(K, L)$ is the Galois connection corresponding to the context $(O, \varnothing(O), R)$, then there exists a matroid $M$ on $O$ with $\sigma$ as its closure operator such that $\sigma$ is the closure operator $L K$.

Proof Definition 1(1) indicates that for any $X \subseteq O$, there are $K(X)=\{Z \subseteq O \mid \forall x \in$ 
$X,(x, Z) \in R\}$ and $L(K(X))=\{a \in O \mid \forall Z \in K(X),(a, Z) \in R\}$.

Since $\sigma(\sigma(X))=\sigma(X)$ holds by (s3). In addition, there is $\rho(\sigma(X) \cup x)=\rho(X \cup X)=\rho(X)=\rho(\sigma(X))$ for any $x \in X$. These cause $\sigma(X) \in K(X)$. For $a \in L K(X), \sigma(X) \in K(X)$ follows $(a, \sigma(X)) \in R$. By the definition of $R$, (I), (II) and (IV), we obtain $\rho(X \cup a)=\rho(\sigma(X) \cup a)=\rho(\sigma(X))=\rho(X)$. Hence, $a \in \sigma(X)$ holds. This implies $L K(X) \subseteq \sigma(X)$.

On the other hand, for any $Z \in K(X)$, there are $\rho(Z \cup x)=\rho(Z)$ and $\sigma(Z)=Z$ for every $x \in X$. In light of Definition 3, $x \in \sigma(Z)$ is correct. Furthermore, we find $X \subseteq Z$. So, $\sigma(X) \subseteq Z$ holds. We may confirm $\rho(Z \cup d)=\rho(Z)$ for each $d \in \sigma(X)$. Thus, $(d, \sigma(X)) \in R$ is true. This follows $\sigma(X) \subseteq L K(X)$.

Therefore, we receive $L K(X)=\sigma(X)$ for any $X \subseteq O$. This means $\sigma=L K$.

Theorem 2 Let $R \subseteq O \times \varnothing(O)$ be established as:

for $x \in O$ and $Y \subseteq O,(x, Y) \in R \Leftrightarrow$ there is $I \subseteq Y$ satisfying $\rho(I \cup x)=|I|=\rho(Y)$.

If $(K, L)$ is the Galois connection corresponding to $(O, P(O), R)$. Then $\sigma=L K$ is correct.

Proof According to Definition 1, we firmly believe $K(X)=\{Z \subseteq O \mid \forall x \in X,(x, Z) \in$

$R\}$ and $L(K(X))=\{a \in O \mid \forall Z \in K(X),(a, Z) \in R\}$.

Let $x \in X$ and $Z \in K(X)$. By (III), (VII) and Lemma 3, $(x, Z) \in R$ determines $\rho\left(I_{x} \cup x\right)=$ $\left|I_{X}\right|=\rho(Z)$ for some $I_{x} \subseteq Z$. Considering the matroid's properties in [2] with (III) and (IV), we assure $I_{X}$ to be a base in $Z$. Thus, $x \in \sigma\left(I_{X}\right) \subseteq \sigma(Z)$ is right. So, $X \subseteq \sigma(Z)$ holds. Furthermore, there is $\sigma(X) \subseteq \sigma(Z)$. Additionally, in view of the maximal independent property of $I_{x}$ in $Z$, (IV) and (VII), we may state that $\rho\left(I_{\mathcal{X}} \cup x\right)=\left|I_{\mathcal{X}}\right|=\rho(Z)=\rho(\sigma(Z))$ holds and $I_{\mathcal{X}}$ is a base of $\sigma(Z)$. Moreover, we obtain $\sigma(Z) \in K(X)$. We may obtain easily that for any $x \in X$, there is $(x, X) \in R$. This carries out $X \in K(X)$. Therefore, $\sigma(X) \in K(X)$ follows.

Since for any $a \in L K(X)$, there exists $(a, Z) \in R$ for each $Z \in K(X)$. Recalling (IV)

and Definition 3, we may easily demonstrate $a \in \sigma(Z)$. Moreover, we confirm $L(K(X))=\cap Z \in K(X) \sigma(Z$ )$=\sigma(X)$

Summing up, $L K=\sigma$.

Theorem 3 Let $R \subseteq O \times \varnothing(O)$ be described as:

for $x \in O$ and $Y \subseteq O,(x, Y) \in R \Leftrightarrow \sigma(Y \cup x)=\sigma(Y)$.

If $(K, L)$ is the Galois connection generated by $(O, P(O), R)$, then $\sigma$ is properly $L K$.

Proof According to Definition 1, (v1) and (v2), we may indicate that for $X \subseteq O$, there are $K(X)=$ $\{Z \subseteq O \mid \forall x \in X,(x, Z) \in R\}$ and $L(K(X))=\{a \in O \mid \forall Z \in K(X),(a, Z) \in R\}$.

For $x \in X$ and $Z \in K(X)$, in light of $(x, Z) \in R$ and (V), we confirm $x \in \sigma(Z)$. This follows $X \subseteq \sigma(Z$ ) for $Z \in K(X)$. Thus, we receive $\sigma(X) \subseteq \sigma(\sigma(Z))=\sigma(Z)$. Additionally, $(x, X) \in R$ is obvious. This determines $X \in K(X)$ and $x \in \sigma(X)$. Considering this result with (VI), we may be assured that $\sigma(\sigma(X) \cup x)=\sigma(X \cup x)=\sigma(X)=\sigma(\sigma(X))$ is true. So $\sigma(X) \in K(X)$ is followed.

By (V) in Section 2, for any $Z \in K(X), a \in L K(X)$ will cause $a \in \sigma(Z)$. Hence, it follows $a \in \sigma(X$ ). However, for each $b \in \sigma(X)$, we obtain $b \in \sigma(Z)$ since $\sigma(X) \subseteq \sigma(Z)$ for any $Z \in K(X)$. Therefore, we demonstrate $\sigma(\sigma(Z) \cup b)=\sigma(\sigma(Z))=\sigma(Z)$. Furthermore, by definition of $R$ and (VI), we may gain $\sigma(Z)=\sigma(Z \cup b)$. Finally, there is $b \in L K(X)$.

Adding up, we may express $L K=\sigma$.

Theorem $4 \mathrm{Let} \mathscr{H} M$ be the set of hyperplanes of $M$. Let $R \subseteq O \times \gamma(O)$ be defined as for $x \in O$ and $Y$ $\subseteq O,(x, Y) \in R \Leftrightarrow x \in Y$ and there is $H \in \mathcal{H} M$ satisfying $Y=H$.

If $(K, L)$ is the Galois connection generated by $(O, \varnothing(O), R)$. Then, $\sigma=L K$ is correct.

Proof Divided two parts to finish the proof.

(Part 1) This part is to prove that for any $F \in \mathscr{F}$, it has $F=\cap F \subseteq H \in \mathscr{Y} M H$.

We firstly prove: let $L$ be a geometric lattice with $H=\left\{H_{1}, \ldots, H_{n}\right\}$ as the coatoms of

$L$ and let $A \in L \backslash\{1\}, A \leq H_{1}, \ldots, H_{m}$ and $A \not H_{j}$ for any $H_{j} \in \mathcal{H} \backslash\left\{H_{1}, \ldots, H_{m}\right\}$. Then we assert $A=\wedge_{i=1}^{m} H$

Obviously, $A \leq \Lambda_{i=1}^{m} H_{i \cdot i}$ is right

We will prove by induction on $h(1)$ (i.e., on $h(L)$ ) to show our assertion. 
If $h(1)=1$. Then the need follows evidently.

If $h(1)=2$.

The need result is followed because on this occasion it exists one and only one geometric lattice $L=\{0$, $\left.1, x_{i},(i=1, \ldots, n \geq 2)\right\}$ where $x_{i}$ satisfies $0<x_{i}{ }_{i=1}$ and $x_{i}$ is parallel with $x_{j}$ for any $i=j,(i, j=1$, $\ldots, n)$. We may state easily that $\left\{x_{i}: i=1, \ldots, n\right\}$ is the coatoms of $L$, and besides, $0=\wedge_{i=1}^{n} x_{i}=$ $\wedge_{i=1}^{n} x_{i}$ for any $1<s \leq n$. Furthermore, $[A, 1]$ is geometric by Lemma 2(2). This determines that if $A$ $<\wedge_{i=1}^{m} x_{i}$, then under this occasion, $[A, 1]$ is not geometric according to the non-existence of $A$. In other words, $A=\wedge_{i=1}^{m} H_{i}$ is true.

Suppose for $h(L)<s$, the need result is correct.

Now let $L$ be geometric and $h(L)=s$.

Let $A \in L \backslash\{0\}$ and $A \leq H_{i},(i=1, \ldots, m)$ but $A \$ H_{j} \in \mathscr{H} \backslash H_{i},(i=1, \ldots, n ; j \in$ $\{1, \ldots, n\} \backslash\{1, \ldots, m\})$. It is evident $h(A) \geq 1$. In addition, $[A, 1]$ is geometric owing to Lemma $2(2)$, and $\left\{H_{1}, \ldots, H_{m}\right\}$ is also the set of coatoms in $[A, 1]$. By the induction, we obtain $A$ $=\wedge_{i=1}^{m} H_{i}$. Recalling the selection of $A, A=\wedge_{i=1}^{m} H_{i}$ is true in $L$. Especially, if we select $A$ as an atom in $L$, then we may receive $A=a_{t}=\wedge_{i=1}^{t m} H_{i}$ where $a_{t} \leq H_{i}$ and

$a_{t} \not H_{j},\left(i=1, \ldots, t_{m} ; j \in\{1, \ldots, n\} \backslash\left\{1, \ldots, t_{m}\right\}\right)$. Because $0=\wedge_{t \in \tau} a_{t}$, where every $a_{t}$ is an atom $(t \in \mathcal{T})$. This causes $0=\Lambda_{i=1}^{n} H_{i}$. Hence, in a geometric lattice, every element is a meet of some of coatoms.

Secondly, because of Lemma $3,(\mathscr{F}, \subseteq)$ is geometric with $\mathscr{H} M$ as its set of coatoms. By the above discussion, there exist $H_{i} \in \mathcal{H}_{M},\left(i \in \phi_{\mathcal{O}} F\right)$ satisfying $F=\cap_{F \subseteq H i \text {, and } i \in \oplus_{\phi F}} H_{i}$ and $F \Varangle H_{j},\left(H_{j} \in \mathcal{L}_{M} \backslash\right.$ $\left.\left\{H_{i}: i \in \phi_{F}\right\}\right)$.

(Part 2) This part is to prove $\sigma=L K$.

In virtue of the definition of $\mathscr{H}_{M}$, we assure $\mathscr{H}_{M} \subseteq \mathscr{F}$. In view of Lemma 3(3), $\cap_{\alpha \in{ }_{\alpha}} H_{\alpha} \in \mathscr{F}$ is correct, where $H_{\alpha} \in \mathcal{H}_{M}(\alpha \in \phi)$. Since for $X \subseteq O$, there are $K(X)=\{Z \subseteq O \mid \forall x \in X,(x, Z) \in$ $R\}$ and $L(K(X))=\{a \in O \mid \forall Z \in K(X),(a, Z) \in R\}$. For any $Z \in K(X),(x, Z) \in R$ will carry out $Z \in \mathscr{H} M$ and $x \in Z$. This decides $X \subseteq Z$ and $K(X) \subseteq \mathscr{H} M$. Additionally, for every $H$ $\in \mathcal{H} M$ and $X \subseteq H$, it causes $H \in K(X)$. Hence,by (Part 1), $\sigma(X)=\cap_{Z \in K(X)} Z$ is followed.

In the following, we consider the properties of $L K(X)$.

For $a \in L K(X)$, there exists $(a, Z) \in R$ for any $Z \in K(X)$. Thus, it follows $a \in Z$.

Hence, $L K(X) \subseteq Z$ holds for any $Z \in K(X)$. Furthermore, $L K(X) \subseteq \square \cap Z \in K(X){ }_{Z} Z$ is true. Considering $\sigma(X)=$ $\cap_{Z \in K(X)} Z$ with $L K(X) \subseteq \cap_{Z \in K(X)} Z$, we may obtain $L K(X) \subseteq \sigma(X)$. Conversely, $b \in \sigma(X)$ points out $b \in$ $\cap_{Z \in K(X)} Z$. Thus, $b \in Z$ is real for any $Z \in K(X)$.

We may express $(b, Z) \in R$. Moreover, $b \in L K(X)$ is correct. Therefore $\sigma(X) \subseteq L K(X)$.

Hence $L K=\sigma$ holds.

Theorem 5 Let $M$ be a matroid defined on $O$ with $\sigma$ as its closure operator and $\mathscr{H} M$ as its the set of hyperplanes. If $\sigma={ }^{\prime \prime}$ where "'is the Galois connection of a context $(G, P, I)$. Then $\mathcal{H}$ is $\mathcal{H} M$ where $\mathcal{H}$ $=\left\{X \subseteq G \mid X^{\prime \prime \prime}=X\right.$, there exists a set $X$ covered by the greatest element of $\left.\operatorname{Gal}(G, P, I)\right\}$.

Next we analyze the differences and links among the contexts provided in the above Theorems and point out some of their significance. Let $R_{j}$ be the relation defined in Theorem $j,(j=1,2,3,4)$ and $\mathcal{H}_{M}$ be the set of hyperplanes of $M$.

(3.1) $(x, Y) \in R_{2} \Leftrightarrow \rho(I \cup x)=|I|=\rho(Y)$ for some $I \subseteq Y .|I|=\rho(Y)$ causes the base property of $I$ in $Y$. Considering with (I)-(VII), we may point out: $(x, Y) \in R 2 \Leftrightarrow \rho(I \cup x)=|I|=\rho(Y)=\rho(Y \cup x) \Leftrightarrow x$ $\in \sigma(I \cup x)=\sigma(Y \cup x) \Leftrightarrow \sigma(Y \cup x)=\sigma(Y) \Leftrightarrow(x, Y) \in R_{3}$.

Thus, there is $\left(O, \varnothing(O), R_{2}\right)=\left(O, \varnothing(O), R_{3}\right)$.

(3.2) By (I)-(VII), if $(x, Y) \in R 1$, then $x \in \sigma(Y)$.

If $(x, Y) \in R_{3}$, then $x \in \sigma(Y)$. But $(x, Y) \in R_{3}$ may not follow $Y=\sigma(Y)$. 
Therefore $(x, Y) \in R_{1} \Rightarrow(x, Y) \in R_{3}$, and not vice versa. In other words, the context appeared in Theorem 1 is different from the context defined in Theorem 3.

(3.3) $(x, Y) \in R 4 \Leftrightarrow$ " $Y \in \mathcal{K}_{M}$ and $\sigma(Y \cup x)=\sigma(Y) " \Leftrightarrow " Y \in \mathcal{K}_{M}$ and $\rho(Y \cup x)=\rho(Y)$ " $\Leftrightarrow$ " $Y$ $\in \mathcal{H}_{M}$ and $\sigma(Y \cup x)=\sigma(Y)=Y " \Rightarrow(x, Y) \in R_{1}$, but not vice versa.

$(x, Y) \in R 4 \Leftrightarrow$ " $Y \in \mathcal{H} M$ and $x \in Y$ " $\Leftrightarrow$ " $Y \in \mathcal{H} M$ and $\rho(Y \cup x)=\rho(Y)$ " $\Leftrightarrow$ "for any base $I Y$ of $Y$, $\rho(Y)=\rho(I Y \cup x)=|I Y|$ and $Y \in \mathscr{L}(M " \Rightarrow(x, Y) \in R 2$, but not vice versa.

The analysis with (3.1) taken together express that the context provided in Theorem 4 is not the same to that in Theorem $\mathrm{j},(j=1,2,3)$.

(3.4) Though the context in Theorem 2 is the same to that in Theorem 3 , they use the different words to state the same context. This is a good way for the users because users can get more chances to select the best description for their topics.

It is valuable to note that in linear optimization, many people like the greedy algorithm. In [2,pp.357360], it points out that we may utilize greedy algorithm to characterize a matroid. If we describe a context by greedy algorithm, the theory of contexts (or say, concept lattice theory) will be much more blooming in optimization, and vice versa.

Additionally, we may know that a matroid links with a geometric lattice (cf.[2,Chapter

3]). If a context is derived from a matroid with the standard language of lattice theory, then it will cause a new connection between matroid theory and concept lattice theory. Actually, under isomorphism, utilizing the above idea, some of works in [21] has done in this field.

(3.5) We may express according to $[9,10,22]$ that a main interest in data analysis and formal concept analysis is to reveal and describe structure of empirical data. U.Wille in [23] desires to represent empirical data by matroids and presents the definition of repre-sentability of data by a matroid. However, U.Wille in [23] instructs that there is no simple characterization of the set structures that admit matroid constructions, one should try to search matroid representations from an algorithmic point of view. Theorem 4 provides a context with the family of hyperplane sets of a matroid, and meanwhile, the definition of representability of data by a matroid is also relative with the hyperplanes of a matroid. What is the relationship between the context in Theorem 4 and the context discussed for its representability in [23]? It is kept for our future research.

(3.6) (3.4) and (3.5) not only state the important for a context provided by a matroid such as the above four Theorems, but also describe the significance to express the same context with different ways such as Theorem 2 and Theorem 3. In the future, we may hope to produce much more contexts relative to matroids in order to satisfy our discussion.

(3.7) $\mathrm{Li}$ et al. [24] point the relationships between rough sets and matroids with lattice theoretic ideas. $\operatorname{Gal}(O, P, R)$ is a lattice. Hence, using rough set to find a context by amatory is a good idea for our future research.

We may believe that there are much better ways to calculate the closed sets of a matroid from its hyperplanes. But we may believe that our way here is a new idea. We may hope that in the future, there are much more new ideas to up to the user's attempt.

\section{Applications}

In Section 3, it presents some contexts relative to a matroid. We will introduce the ir some applications in mathematical opinions, particularly, in using these contexts with ready- made algorithms for constructing concept lattices to construct the closed sets of a matroid.

By (v2), for a context $(O, P, R)$ provided in Section 3 , if $(K, L)$ is the corresponding Galois connection, then $\Theta(L K)=B O$.

If $(O, P, R)$ is a context and $M$ is relative to $(O, P, R)$ as that in Theorem $j,(j \in\{1,2,3,4\})$, then $\mathcal{F}=\mathscr{B}(L K)$, where $F$ is the class of the closed sets of $M$.

In light of [9,10,13-18,22], there are many ready-made algorithms building up concept lattices and also searching out extent lattices. We can choose any of these ready-made algorithms especially the algorithms for searching out extent lattices to find out $\mathscr{F}$. Hence, we may state that the question raised in (1.5) is answered here.

In [2, Chapter 2], it introduces the properties about the dual $M^{*}$ of $M$. (The definition of $M^{*}$ is seen [2,p.34]). We may notice that until now, based on our knowledge, it does not have an algorithm to yield out the construction of $M^{*}$ from $\mathscr{F}$ directly. Next, we may build up $M^{*}$ correctly by selecting a top- 
down (from the maximal extent to the minimal one) approach of constructing an extent lattice or a concept lattice. The reason is the following (4.1)-(4.4):

(4.1) [2,p.39,Theorem 2] describes that a set $C$ is a circuit of $M^{*}$ if and only if its complement $O \backslash$ $C$ is a hyperplane of $M$ on $O$.

(4.2) [2,p.8, Theorem 5] indicates that a matroid is uniquely determined by its set of circuits.

(4.3) [2,pp.48-49] points out that an element in $(\mathscr{F}, \subseteq)$ is covered by $O$ if and only if it is a hyperplane of $M$.

(4.4) By (4.1)-(4.4), we may describe that using a top-down method for searching out a concept lattice, we may directly find out all the hyperplanes of $M$, i.e. the set of covered elements by the maximum member in $\mathcal{B}(L K)$. That is to say, until now, we may obtain many algorithms to build up the dual of $M$.

In [15], it points out that different algorithms play differently on different databases (or say, contexts). Sometimes authors compare their algorithms with others on specific data sets. We may propose that the community should reach a consensus with respect to databases to be used as test beds. For the reasons mentioned above, we may not indicate that one of contexts in the four Theorems in Section 3 is better than the others. If we consider a solution of a question relative to a matroid, then we may be better to choose a context based on our databases to carry out the solution.

Now, in Section 3, we find out four contexts relative to $M$ though actually they are said to be three. These contexts can be used to search out $\mathscr{F}$, and meanwhile, build up $M^{*}$. Certainly, these contexts may be applied in the other fields. We may believe that it will discover many contexts relative to a matroid because there are dozens of equivalent ways

To define a matroid. These are left rooms for the future.

\section{References}

[1]. Whitney H. On the abstract properties of linear dependence. American Journal of Math- ematics, 1935, 57(3), 509-533.

[2]. Welsh D.J.A. Matroid Theory. London: Academic Press, 1976.

[3]. Hliněný P. A parametrized algorithm for matorid branch-width. SIAM Journal on Com- puting, 2005, 35(2), $259-277$.

[4]. Gabow H.N., Westermann H.H., Forests, frames, and games: algorithms for matroid sums and applications. Algorithmica, 1992, 7, 465-497.

[5]. Mao H. On closure axioms for a matroid using Galois connections. Mathematical Com- munications, 14(2)(2009), 425432.

[6]. Ganter B., Reuter K. finding all closed sets: a general approach. Order, 1991, 8, 283- 290.

[7]. Wille R. Restructuring lattice theory: an approach based on hierarchies of concepts, in Rival I.(ed.) Ordered sets. Dordrecht-Boston: Reidel, 1982, 445-470.

[8]. Birkhoff G. Lattice Theory, 3rd.ed. Providence: Academican Math. Soci., 1979.

[9]. Ganter B., Wille R. Formal Concept Analysis: Mathematical Foundations. Heidelberg: Springer-Verlage Berlin, 1999.

[10]. Davey B.A., Priestley H.A. Introduction to Lattices and Order, 2nd. ed. Cambridge: Cambridge University Press, 2003.

[11]. Driessel K.R. Closure operators and Galois connections. Date: http://homepage.mac.com/driessel/IMA/realalg/closure.pdf

[12]. Denecke K., Erné M., Wismath S.L. Galois Connections and Applications. Dordrecht: Kluwer Academic Publishers, 2004.

[13]. Prediger S. Formal concept analysis for general objects. Discrete Applied Mathematics, 2003, 127, $337-355$.

[14]. Berry A., Bordat J., Sigayret A. A local approach to concept generation. Annals of Mathematics and Artificial Intelligence, 2007, 49, 117-136.

[15]. Choi V. Fasteralgorithms for constructing a concept(Galois) lattices. arXiv:cs.DM/0602069 v2 1 June 2006.

[16]. Kuznetsov S., Obiedkov S. Comparing performance of algorithms for generating con- cept lattices. Journal of Experimental \& Theoretical Artificial Intelligence, 2002, 14, 189-216.

[17]. Nourine L., Raynaud O. A fast algorithm for building lattices. Information Processing Letters, 1999, 71, 199-204.

[18]. Belohlávek R., Bates B.D. et al. Characterizing trees in concept lattices. International Journal of Uncertainty, Fuzziness and Knowledge-Based Systems, 2008, 16(1), 1-15.

[19]. Mao H. An approach to access to a concept lattice via the idea of lattice theory, Uni-versity Politehnica of Bucharest Scientific Bulletin, series A, Mathematics and Physics, 2011, 73(2), 37-50.

[20]. Hitzler P., Krötzsch M., Zhang G.-Q. A categorical view on algebraic lattices in formal concept analysis, Fundamenta Informaticae, 2006, 74, 1-29.

[21]. Mao H. On lattice-theoretical construction of matroids. Analele Stiintifice ale Univer- sitatii "AI.I.Cuza" din Lasi Matematica, 2013, 59(1), 201-208.

[22]. Carineto C., Romano G. Concept Data Analysis: Theory and Applications. England: John Wiley \& Sons Ltd., 2004.

[23]. Wille U. On extending closure systems to matroids. European Journal of Combina- torics, 2002, 23, 131-139.

[24]. Li X., Yi H., Liu S. Rough sets and matroids from a lattice-theoretic viewpoint, Infor-mation Sciences, $2016,342,37-52$. 